ISSN 1794-9831

E-ISSN 2322-7028 Vol. 12 No. 1

Ene - Jun 2015

Cúcuta, Colombia

Recibido:

19 de Septiembre

de 2014

Aprobado:

9 de Marzo

de 2015

* Enfermera Especialista en Educación Sexual y Especialista en Prácticas Pedagógicas Universitarias. Docente.

Universidad Francisco de Paula Santander. Correo electrónico: pavelapimo@ hotmail.com

** Enfermera. Caja de Compensación Familiar del

Oriente -

Comfaoriente.

Correo electrónico: yeyekar 13@ hotmail.com

*** Enfermera. Corporación IPS

Norte de Santander. Correo electrónico: ayita-10@hotmail. com

**** Descriptores en Ciencias de la Salud (DeCS), en la página http:// decs.bvs.br/E/ homepagee.htm de la Biblioteca virtual en salud del proyecto BIREME, de la Organización Mundial de la Salud y de la Organización Panamericana de Salud.

\section{Actitudes y prácticas frente a la sexualidad de los homosexuales}

\author{
Patricia Vélez Laguado* \\ Yessenia Rojas Dodino** \\ Andrea Marcela Mogollón González ${ }^{* * *}$
}

\section{RESUMEN}

Objetivo: evaluar las actitudes y prácticas frente a la sexualidad de la comunidad de lesbianas, gais, bisexuales, transexuales, intersexuales y como estas influyen en su salud sexual y reproductiva. Materiales y Métodos: estudio cuantitativo, descriptivo de corte transversal. La población estudio fueron 335 homosexuales pertenecientes a una fundación X, la muestra correspondió a 180, a quienes se aplico el instrumento Actitudes y Prácticas frente a la Sexualidad de los Homosexuales. Los criterios de inclusión fueron ser homosexual ser mayor de 18 años, haber tenido experiencias sexuales homosexuales, no presentar enfermedades mentales y participar voluntariamente. Resultados: predominaron los gais en un $50,5 \%$, el estado civil preponderante fue soltero con un $70,6 \%$; la edad predominante fue 24 a 29 años, y el nivel educativo más frecuente fue secundaria completa $27,7 \%$; en cuanto a información de experiencias sexuales la primera experiencia física fue sexo con 52,2\%, el 61,1\% inició su vida sexual entre los 14 y 19 años; el 43,9\% ha tenido de 11 a 20 compañeros sexuales, y en los últimos 6 meses el $65 \%$ ha tenido relaciones sexuales ocasionales, en actitudes el $43,9 \%$ dio a conocer su orientación sexual a amigos. En prácticas el 58,4\% conoce a sus parejas sexuales por amigos; en desarrollo y actividad en salud sexual y reproductiva el $8,9 \%$ ha presentado infecciones de transmisión sexual y ha recibido tratamiento completo, el $56,7 \%$ no utilizó preservativo en su primera relación sexual, y solo el 54,4\% sabe que es el cáncer genital. Conclusión: Según las variables planteadas las actitudes y las prácticas de los homosexuales si afectan su salud sexual y reproductiva.

PALABRAS CLAVE: actitud, salud sexual y reproductiva, sexualidad ${ }^{* * * *}$.

Para citar este artículo / To reference this article / Para citar este artigo

Vélez-Laguado P, Rojas-Dodino Y, Mogollón-González AM. Actitudes y prácticas frente a la sexualidad de los homosexuales. Rev. cienc. cuidad. 2015; 12(1): 40-52. 


\section{Attitudes and practices face to homosexual sexuality}

ISSN 1794-9831

E-ISSN 2322-7028

Vol. 12 No. 1

Ene - Jun 2015

Cúcuta, Colombia

\section{ABSTRACT}

Objective: To test attitudes and practices face to sexuality of the lesbian, gay, bisexual, transgender, intersex community and how they are influencing their sexual and reproductive health. Materials and Methods: Quantitative, descriptive cross-sectional study. The study populationwere 335 homosexual members belonged to $X$ foundation, the sample consisted of 180 people, to the Attitudes And Practices instrument was applied against gay sexuality. Inclusion criteria were: being homosexual older than 18 years old, having homosexual experiences before, haven't suffered mental illness and to participate voluntarily. Results: gays predominated in $50.5 \%$, the predominant marital status was single with $70.6 \%$; the predominant age was 24-29 years and the most common education level was secondary with a $27.7 \%$; as regard information about sexual experiences ; the first physical experience was sex with a $52.2 \%, 61.1 \%$ of population sample began their sexual life between 14 and 19 years old; $43.9 \%$ of them had 11 to 20 sexual partners, and in the last six months $65 \%$ had casual sex. In attitudes $43.9 \%$ disclosed their sexual orientation to friends; in practice $58.4 \%$ met their sexual partners by friends; in development and activity in sexual and reproductive health $8.9 \%$ presented sexually transmitted infections and received full treatment, $56.7 \%$ did not use condoms during their first sexual intercourse, and only $54.4 \%$ know what is a genital cancer. Conclusion: according to the considered variables the attitudes and practices of homosexual people affect their sexual and reproductive health.

KEYWORDS: attitude, sexual and reproductive health, sexuality. 
ISSN 1794-9831

E-ISSN 2322-7028 Vol. 12 No. 1

Ene - Jun 2015

Cúcuta, Colombia

\section{Atitudes e práticas contra a sexualidade gay}

\section{RESUMO}

Objetivo: avaliar as atitudes e práticas em relação à sexualidade de lésbicas, gays, bissexuais, transgêneros, intersex e como você está influenciando a sua saúde sexual e reprodutiva. Materiais e Métodos: estudo transversal, quantitativo, descritivo. A população do estudo eram 335 os membros homossexuais de uma fundação X, a amostra foi composta de 180 critérios, para a Atitudes E Práticas instrumento foi aplicado contra a sexualidade gay. De inclusão foram ser gay ter mais de 18, tiveram experiências sexuais homossexuais, não presente doença mental e participar voluntariamente. Resultados: gays predominou em 50,5\%, o estado civil predominante foi a única com $70,6 \%$; a faixa etária predominante foi de 24-29 anos eo ensino secundário mais comum foi de 27,7\% concluída; informações sobre experiências sexuais primeira experiência sexual física foi de $52,2 \%$, $61,1 \%$ iniciaram a vida sexual entre 14 e 19 anos; $43,9 \%$ tinham de 11 a 20 parceiros sexuais, e nos últimos seis meses $65 \%$ fizeram sexo casual. Em atitudes $43,9 \%$ divulgados sua orientação sexual aos amigos; na prática, 58,4\% sabiam que seus parceiros sexuais por amigos; em desenvolvimento e atividade na área da saúde sexual e reprodutiva de $8,9 \%$ apresentada infecções sexualmente transmissíveis e recebeu tratamento completo, $56,7 \%$ não usaram preservativos na primeira relação sexual, e somente $54,4 \%$ sabem que é cancro genital. Conclusão: de acordo com as variáveis levantadas as atitudes e práticas dos homossexuais, se eles afetam a sua saúde sexual e reprodutiva.

PALAVRAS-CHAVE: atitude, saúde sexual e reprodutiva, sexualidade. 


\section{INTRODUCCIÓN}

$\mathrm{L}$ os homosexuales son un grupo vulnerable que requiere atención en salud específica, aunque en algunos casos estos individuos no acuden al servicio de salud por temor a ser discriminados por el personal de salud debido a su orientación sexual o por como practican su sexualidad; se debe tener en cuenta que el hecho de no acudir genera un impacto negativo en cuanto a la prevención, detección y atención de enfermedades de trasmisión sexual (1).

El no acudir genera un factor de riesgo que afecta la salud sexual y reproductiva, teniendo en cuenta que involucra el bienestar físico -en cuanto existe la posibilidad de adquirir una infección de trasmisión sexual o daños en los órganos sexuales o cualquier parte del cuerpo- y psicológico, centrándose solamente en satisfacer a su pareja y dejando atrás su placer, lo cual conduce al individuo a un daño en su parte emocional (1).

Por lo general los homosexuales, cuando se trata de su salud sexual, tienden a ser reservados con las demás personas, puesto que pueden llegar a sentir un grado de discriminación por sus prácticas o actitudes frente a la sexualidad, inclusive con el personal de salud, debido a que en este ámbito también se encuentra el rechazo (1).

La homosexualidad se considera como la práctica de relaciones eróticas con personas del mismo sexo. En la actualidad se entiende que la homosexualidad es una elección o una orientación natural, sin embargo, varía de acuerdo al país: mientras que en ciertos países los homosexuales pueden contraer matrimonio y adoptar hijos, en otros son perseguidos por su condición sexual y hasta enviados a prisión o condenados a muerte (1).

Uno de los principales problemas que enfrenta un individuo es la definición de su propia sexualidad. En la etapa adolescente llega la experimentación con personas del mismo sexo y esto puede llevarlo a conflictos internos de orientación, problemas de salud, aceptación del entorno, etc., pues una persona puede experimentar prácticas o situaciones sexuales con personas del mismo sexo, pero en realidad no es lo que quiere ni es lo que siente, sólo hace parte de un momento de confusión del que se da cuenta cuando empieza a conseguir su propia identidad sexual (2).
La persona se compromete tanto con su propio comportamiento sexual como con los estímulos que elige teniendo la libertad para determinar su conducta sexual, configurándola y moldeándola según lo que ha elegido y su estilo personal que, a su vez, está en parte determinado por el modo sexual y personal. Las actitudes frente a la sexualidad en homosexuales se ven reflejadas en la conducta, los sentimientos, la atracción y todo aquello que abarca la experiencia (1).

La salud reproductiva es un estado general de bienestar físico, mental y social. Es la capacidad de disfrutar de una vida sexual satisfactoria sin riesgos de procrear y la libertad para decidir hacerlo o no hacerlo; es el conjunto de métodos, técnicas y servicios que contribuyen a la salud y el bienestar sexual para evitar y resolver los problemas relacionados con la salud reproductiva. (2).

Puede ocurrir que los homosexuales se separen de su entorno y presenten problemas de reconocimiento que desarrollen baja autoestima afectando su bienestar mental y, por querer salir de allí, empiecen a realizar actos que no los hagan sentir culpables ni diferentes, solamente sentirse orgullosos de lo que son y, por eso, llevar a cabo su sexualidad de acuerdo con su parecer, Ante los demás, sus prácticas sexuales se consideran indebidas fuera de lo común, no aptas ante Dios, no permitidas y, en ocasiones, como un pecado (3).

Estas prácticas hacen referencia a las diferentes formas de sentir placer, de mostrar sus sentimientos a la pareja o simplemente a situaciones alocadas de momento, las cuales han aumentado la tasa de prevalecía del virus de inmunodeficiencia humana (VIH) y riesgo sexual entre los hombres y mujeres gay, contribuyendo a que las actitudes y prácticas que éstos desarrollan, en muchas ocasiones estén altamente involucradas con afectar su salud sexual y reproductiva (3)

La práctica de relaciones anales, relaciones orales, restregarse los cuerpos con heces, realizar prácticas sadomasoquistas, fisting, uso de enemas o sexo con animales, entre otras, en ocasiones trae consecuencias desfavorables que conllevan a no disfrutar totalmente de su sexualidad; sin embargo, se puede decir que los homosexuales hacen el amor de una forma parecida a como lo hacen los heterosexuales (3).

Los resultados del estudio pretenden evidenciar cómo se ve afectada la salud sexual y reproductiva 
ISSN 1794-9831

E-ISSN 2322-7028

Vol. 12 No. 1

Ene - Jun 2015

Cúcuta, Colombia de los homosexuales y determinar si sus actitudes y prácticas se convierten en un factor de riesgo relevante para la afección de su salud sexual y reproductiva, destacándose como una problemática frecuente en la sociedad, pero pocas veces atendida de la mejor manera por la estigmatización con la que es tratado este grupo en general. Se espera cambiar las ideas erróneas de las personas, lo cual contribuirá a mejorar la calidad de vida de la comunidad homosexual.

\section{MATERIALES Y MÉTODOS}

La investigación es de naturaleza cuantitativa, de tipo descriptivo y corte transversal. La población objeto de estudio fueron 335 homosexuales pertenecientes a una fundación $\mathrm{X}$ de la comunidad de lesbianas, gais, bisexuales, transexuales e intersexuales.

El muestreo fue de tipo probabilístico, aleatorio simple con un cálculo que evidencia un margen de error de $5 \%$, nivel de confianza del $95 \%$ y probabilidad de ocurrencia del $50 \%$, obteniendo así una muestra de 180 individuos.

Se aplico el instrumento Actitudes y Prácticas frente a la Sexualidad de los homosexuales en el primer semestre de 2014; creado por las mismas investigadoras. Se realizó la validación del mismo mediante consulta a tres expertos, quienes hicieron las correcciones pertinentes. En cuanto al análisis de fiabilidad, mediante Alfa de Cronbach, se determinó un coeficiente $\alpha=0.81$, de lo cual se concluyó que la consistencia interna del instrumento era buena; seguidamente se dio paso a la prueba piloto, la cual se realizó con el $10 \%$ de la población objeto de estudio.

Los cambios se hicieron en 5 preguntas de las 50 en total. En lo demás no hubo problemas de comprensión del instrumento ni en otras opciones de respuesta. El grupo manifestó que las preguntas eran claras y no afectaban en ningún sentido a los encuestados.

En cuanto al instrumento y variables, el cuestionario fue dividido en 5 partes: la primera constaba de 6 preguntas relacionadas con las características sociodemográficas del encuestado, la segunda estaba integrada por 8 preguntas relacionadas coninformación y experiencia sexual, la tercera contenía 5 preguntas e indagaba por las actitudes, la cuarta parte involucraba 18 preguntas relacionadas con las prácticas, mientras que la quinta y última parte referenciaba 13 preguntas relacionadas con el desarrollo y la actividad en salud sexual y reproductiva.

La investigación se realizó teniendo en cuenta las normas éticas para la investigación en seres humanos, según Resolución No 008430 del 4 de Octubre de 1993 (4), garantizando la total privacidad y no divulgación de los resultados con nombre propio, debido a que se analizaron de forma general, teniendo en cuenta los principios éticos de privacidad, autonomía, beneficencia y no maleficencia.

En relación con la participación de los investigados, se suministró consentimiento informado escrito, en el cual es importante la comprensión y aclaración del proceso de investigación y cualquier inquietud respecto al propósito, razones y beneficios de la investigación.

\section{OBJETIVOS}

\section{Objetivo general}

Determinar las actitudes y prácticas frente a la sexualidad de los homosexuales.

\section{Objetivos específicos}

- Caracterizar sociodemográficamente la población objeto del estudio.

- Describir la información de experiencias de los homosexuales.

- Identificar las actitudes frente a la sexualidad de los homosexuales.

- Identificar las prácticas frente a la sexualidad de los homosexuales.

- Identificar el desarrollo y la actividad en salud sexual y reproductiva de los homosexuales. 


\section{RESULTADOS Y DISCUSIÓN}

\section{Datos sociodemográficos}

Figura 1. Distribución por orientación sexual de los homosexuales.

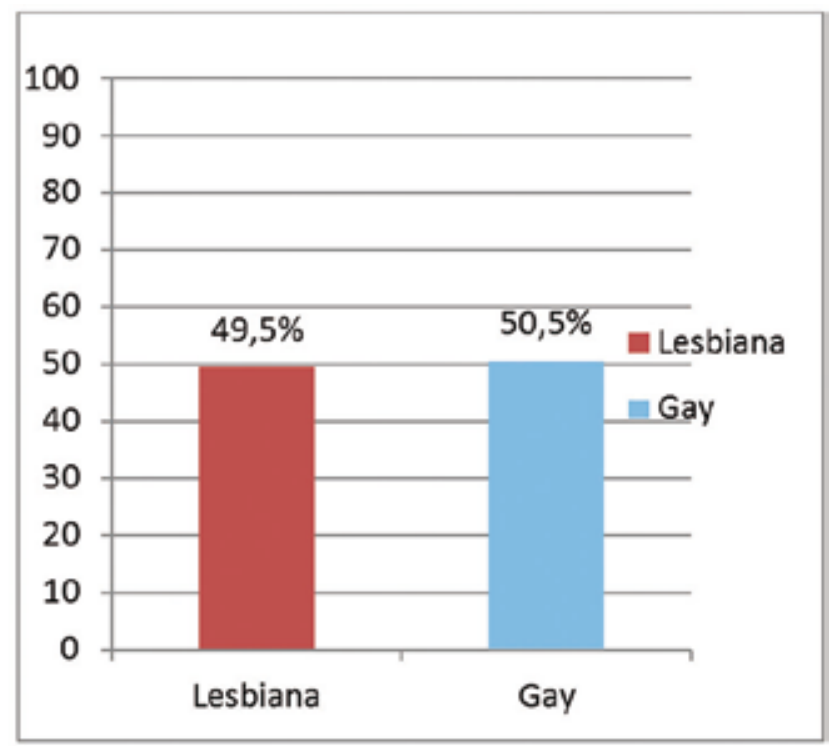

Fuente: Instrumento Actitudes y Prácticas frente a la Sexualidad de los homosexuales, 2014.

Los resultados muestran que el $50,51 \%$ de la población estudiada pertenece a la orientación sexual gay y un $49,5 \%$ a la orientación sexual lesbiana, como se puede a preciar en la figura 1. Velásquez y Toro (5), indican que la "orientación sexual es aquella cuyo amor, deseo o erotismo está focalizado hacia personas de igual sexo, indicadas por el deseo natural tanto sexual como amoroso y erótico hacia personas de igual o distinto sexo".

Estos resultados son similares a la investigación realizada por Moreno (6) en Perú, en donde predominaron los gais en un $53,2 \%$ y las lesbianas en un $46,8 \%$, haciéndose evidente el dominio de la orientación sexual gay más que el de lesbiana, sin una diferencia significativa.

En cuanto al rango de edad que se manejó en la investigación, de 18 a 42 años en adelante, se evidenció que el grupo de mayor representatividad fue el de 24-29 años con un 40,6\%, mientras que el 4,4\% correspondió al rango de 36-41 años. Se evidenció que la población que más se intervino fue la de adulto joven, hallándose similitud con el estudio realizado por Moreno (6), en donde los entrevistados que más predominaron pertenecían al rango de edad entre los 17 a 32 años.

El estudio realizado por la Federación Estatal de Lesbianas, Gais, Transexuales y Bisexuales y Durex (7), muestra en cambio diferencias, pues evidencia que el grupo con mayor representatividad fue de 30 a 39 años, con un $27,6 \%$, que representa a los adultos mayores.

Al evaluar el estado civil de los encuestados se puede evidenciar que predominó en un $70,6 \%$ el de solteros, seguido de un $8,8 \%$ en unión libre y un $0 \%$ viudo, guardando similitud con el estudio realizado por Ortiz y García (8), donde el estado civil que predominó fue soltero sin pareja a diferencia de $12,1 \%$ con pareja y cohabitan, debido que al estar solteros pueden tener más libertad $\mathrm{y}$, con ello, poder conocer más gente y disfrutar de más experiencias sin ningún compromiso.

En cuanto al nivel educativo de los encuestados, los resultados mostraron que un $27,7 \%$ de los homosexuales participantes en el estudio tiene secundaria completa, un $3,9 \%$ posee primaria completa, al igual que primaria incompleta en un 3,9\%, encontrando similitud con el trabajo de Moreno (6), en el cual los homosexuales encuestados presentan, en su mayoría, un 39,2\% secundaria completa. De igual manera, en el estudio realizado por la Federación Estatal de Lesbianas, Gais, Transexuales y Bisexuales y Durex (7) se evidenció con mayor representatividad la educación secundaria completa, con $35,9 \%$, lo que muestra que, en su mayoría, los encuestados no llegan a un mayor nivel educativo, lo cual puede ver afectada su salud sexual y reproductiva en cuanto a sus prácticas y actitudes sexuales.

En cambio, la investigación realizada por Cañejas et al. (9) muestra que de los 157 encuestados, el 85\% había terminado estudios medios o superiores, lo cual permite inferir que el nivel educativo no es un factor concluyente para la realización de prácticas sexuales en los homosexuales, que afecte su salud sexual y reproductiva.

\section{Información y experiencia sexual}

En esta categoría se evidencia que, en los encuestados, la primera experiencia física con una persona de su misma orientación sexual fue sexo con un $52,2 \%$ a diferencia de un $5 \%$ correspondiente a masturbación. 
ISSN 1794-9831

E-ISSN 2322-7028

Vol. 12 No. 1

Ene - Jun 2015

Cúcuta, Colombia
De acuerdo con lo manifestado por los encuestados, en algunas ocasiones la primera experiencia sexual es muy importante y especial porque marca el inicio de una nueva etapa en la vida; pero tomar la decisión de dar este paso no es fácil, las dudas, los temores o la ansiedad pueden estar presentes y llegar a confundir este tipo de situaciones. Para los homosexuales se hace común que el primer encuentro físico con una pareja sea de tipo sexual, sin antes haber pasado por contactos mínimos sin intimidad (6).

De igual manera, en la investigación realizada por Moreno (6) se evidencia que las relaciones sexuales o experiencias coitales ha sido una de las conductas riesgosas más evaluada en la población homosexual, debido a las repercusiones que ha tenido en la salud pública donde predomina, encontrándose que en las lesbianas fue entre los 14 y 19 años en un $90,7 \%$, y en los gays entre los 10 y 14 años en un $67,3 \%$, es decir, en pleno inicio de la adolescencia.

Alcock, de acuerdo a Moral (10), sostiene que "la mayor prevalencia de las conductas sexuales de los homosexuales no sería tanto un gen especifico sino el mayor impulso sexual, determinado por la genética de su sexualidad para abarcar las prácticas sexuales tales como la masturbación, las caricias o el sexo".

Así mismo, al evaluar la edad de la primera relación sexual, se encontró que los rangos 14 a 19 años ocuparon el $61,1 \%$, a diferencia de los 2 rangos de edad de 30 a 39 años y mayor de 40 años que obtuvieron un $0 \%$. Estos resultados son similares al estudio de Moreno (6), donde se encontró que la edad predominante de las lesbianas en su primera relación sexual fue entre los 14 y 19 años con el 90,7\% y en los gais entre los 10 y 14 años con un $67,3 \%$, también en pleno inicio de la adolescencia.

La investigación realizado por Cañejas et al. (9) plantea que la edad de la primera relación sexual fue de $\operatorname{los} 16$ a los 20 años, con un 36,0\%, hallándose similitud con la actual investigación, donde las edades predominantes están incluidas hacia este rango.

Se evidencia, entonces, que la edad para el inicio de las relaciones sexuales en la población homosexual se da en su etapa de adolescencia temprana, con el fin de experimentar vivencias de los demás o simplemente por curiosidad hacia las sensaciones relacionadas con su reconocimiento sexual, lo cual afecta su vida sexual en virtud de los peligros y/o consecuencias que esto conlleva. Por otra parte, se puede ver perjudicada su salud sexual y reproductiva debido a que los órganos sexuales, en esta etapa de adolescencia temprana para el acto sexual, pueden verse afectados al no encontrarse desarrollados completamente o por la falta de conocimiento frente a estos temas, lo cual también trae consecuencias para la salud.

Náder et al. (11) plantean que "hay que recordarle a los jóvenes hombres y mujeres que las relaciones producen una serie de consecuencias, no solamente es el placer y gusto, también una de las funciones de la sexualidad es la parte reproductiva. Muchos jóvenes creen que sólo es tener un contacto genital y se olvidan de las consecuencias. Si el sexo no se hace de forma responsable, pueden adquirir alguna enfermedades de transmisión sexual". Por el contrario, el estudio realizado por la Federación Estatal de Lesbianas, Gais, Transexuales y Bisexuales y Durex (7) indica que las edades predominantes en la primera relación sexual fueron de 25 a 29 años, con un 17,5\%.

En cuanto al rango de edad del primer compañero sexual, fue de 20 a 24 años, equivalente al $40 \%$, a diferencia del de mayor de 40 años que fue de un $0 \%$, lo que muestra que la edad predominante encontrada es el adulto joven. Según Irvin e Hidalgo (12), "la mayoría de los homosexuales sostienen relaciones sexuales con parejas de edades contemporáneas aunque, ciertamente, hay quienes tiene preferencia por personas más jóvenes que ellos"

Al evaluar otras experiencias sexuales en relación al número de parejas sexuales que han tenido los homosexuales hasta la actualidad, se encontró un mayor porcentaje en el rango de 1 a 10 parejas con $43,9 \%$, de 11 a 20 parejas con un $27,8 \%$ y más de 40 parejas con un $1,1 \%$ de los encuestados. Aquí existe semejanza con los resultados del estudio de Sexole (13) realizado en 2010, el cual evidencia que en el número de parejas sexuales hasta la actualidad que han tenido los homosexuales predomina de 2 a 5 compañeros sexuales con el 27,6\%, mostrando que el alto número de compañeros sexuales en los individuos es una práctica de riesgo para la salud sexual y reproductiva puesto que se deben tener en cuenta los compañeros que, con anterioridad, se involucraron en la vida de cada individuo. 
En cuanto a las relaciones sexuales con personas ocasionales en los últimos 6 meses se evidenció que el $65 \%$ refirió haber practicado sexo ocasional, mientras que el $35 \%$ respondió que no, evidenciándose el alto grado de promiscuidad, fenómeno que pone en riesgo la salud sexual de los homosexuales debido a sus prácticas y al alto número de compañeros sexuales en tan poco tiempo.

Estos resultados se asemejan a los encontrados en la investigación realizada por Cañejas et al. (9), en donde se evidencia que la aceptación de compañeros sexuales ocasionales en los últimos 3 meses es de 2 a 5 parejas sexuales, equivalente a un $26,9 \%$. La comunidad homosexual tiene un alto índice de prácticas con compañeros sexuales ocasionales, lo cual implica riesgo su salud sexual debido a la posibilidad de adquirir una enfermedad.

Por su parte, el estudio realizado por Federación Estatal de Lesbianas, Gais, Transexuales y Bisexuales y Durex (7), mostró resultados disímiles donde el $56,7 \%$ de los participantes no tenía relaciones ocasionales y el $43,3 \%$ si lo tenían.

Eighner (14) plantea "no veo nada errado en la promiscuidad homosexual. Pienso que es uno de los aspectos más positivos de la vida gay que la gente de circunstancias muy diferentes pueda alcanzar intimidad muy rápidamente".

\section{Actitudes}

En cuanto a quienes fueron las primeras personas que conocieron la orientación sexual de los encuestados, los resultados mostraron que los amigos con el $43,9 \%$, fue la respuesta que obtuvo la mayor participación, mientras que el personal de salud sólo obtuvo un $0 \%$. Se evidencia similitud con la investigación realizada por Moreno (6) donde el 46,7\% informó a un amigo sobre su homosexualidad, "algunos cuentan que fue un proceso muy rápido y sintieron que todo estaba en su lugar. Sin duda, las circunstancias externas influyen en las dificultades de mostrarse abiertamente, puede depender de la edad pero también del lugar donde se resida y trabaje, entre otros factores".

En cuanto al sentimiento que predominó en su primera experiencia sexual, los resultados muestran que la satisfacción tuvo el mayor porcentaje con $24.4 \%$, seguido de pena con un $21,8 \%$ y sentimientos de culpa con el 5\%. Contrastando con el estudio de Moral (10), en este trabajo el $44 \%$ manifestó nunca sentir satisfacción y el $23 \%$ sí pudo sentir satisfacción; en cuanto a sentimientos de culpa, el 57\% nunca sintió culpa y el $16 \%$ algunas veces.

Al indagar sobre la posibilidad de querer volver a tener una experiencia sexual por gusto y/o placer, el $83,3 \%$ respondió que sí, mientras que el $16,7 \%$ manifestó que no, pudiéndose inferir, a partir de estos resultados, que la mayoría de los homosexuales, después de experimentar su primera relación sexual, quisieron volver a tener otra experiencia ya fuera por gusto y/o placer.

Según De la Cruz (15) "en cuanto a la sexualidad en las parejas homosexuales sucede exactamente al igual que las heterosexuales: están constituidas por dos personas con su forma de entender la sexualidad, sus deseos, sus placeres y sus experiencias sexuales, que llevan a que los encuentros siembren en ellos deseos de volver a experimentar placeres, gustos y sentimientos encontrados que se generaron; por cierto, si hubiera que hablar del placer, las claves del placer homosexual se generan de los deseos y las coherencias, es decir, no sólo el orgasmo, sino también las vivencias y las prácticas".

\section{Prácticas}

En cuanto a las prácticas realizadas por los homosexuales, el sexo oral fue la práctica predominante donde el $52,2 \%$ hace sexo oral y le hacen sexo oral, mientras que al 5,6\% no le gusta el sexo oral y, por lo tanto, realizan otro tipo de prácticas.

Estos resultados muestran similitud con los datos del Family Research Institute, Inc. (Instituto de Investigaciones para la Familia) (16) en Washington, donde se evidenció que los homosexuales practican el sexo oral con la mayoría de sus contactos sexuales, el $70 \%$ de la población encuestada manifestó que realiza y le realizan sexo oral, mientras que el $30 \%$ adquiere un rol en el cual siempre le realizan la práctica del sexo oral.

Los gais que llevan a cabo el sexo oral prácticamente están predispuestos a contraer algún tipo de infección, pues en cada práctica consumen microorganismos inapropiados, provenientes de los fluidos corporales que se involucran en esta práctica, siendo esto un

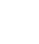


ISSN 1794-9831

E-ISSN 2322-7028

Vol. 12 No. 1

Ene - Jun 2015

Cúcuta, Colombia riesgo para su salud sexual y reproductiva. De igual forma, estas personas pueden adquirir con ello infecciones de transmisión sexual, puesto que muchos de sus encuentros sexuales ocurren con extraños y no con la pareja (16).

De acuerdo con los resultados obtenidos, los homosexuales conocen a sus parejas sexuales a través de amigos en un $58,4 \%$, en discotecas o bares el 16,1\% y en la universidad donde estudia el 4,4\%. Resultados similares se presentan en el estudio realizado por la Federación Estatal de Lesbianas, Gais, Transexuales y Bisexuales y Durex (7) que evidencia que un 74,7 \% conoce a sus parejas sexuales en discotecas o bares, el $31,1 \%$ por amigos y un $7,0 \%$ en el trabajo.

En cuanto a la preferencia de edad de la pareja sexual se pudo evidenciar que el rango de edad de 25 a 29 años predominó en un $43,4 \%$, seguido de 20 a 24 años con un $31,1 \%$ y mayor de 40 años el $0 \%$. El estudio de Gómez (17) afirma que se analizaron las diferencias entre heterosexuales y homosexuales, utilizando los datos de los años 2005 y 2006, encontrando que los varones homosexuales menores de 30 años preferían parejas sexuales unos 4 años mayores que ellos, a partir de los 40 comenzaban a interesarse por parejas con una edad similar a ellos y, a partir de los 50, preferían a parejas 4 o 5 años más jóvenes. Este patrón fue similar al encontrado entre los heterosexuales.

En el mismo estudio, las mujeres de ambas orientaciones sexuales y de todas las edades consideraban adecuadas a parejas sexuales mayores que ellas. Sin embargo, las lesbianas consideran aceptable una pareja más joven a medida que ellas envejecen, de manera similar a como sucede con los varones, pero de un modo menos pronunciado (17).

Resultados similares se encuentran en el estudio de Silverthorne y Quinsey, de acuerdo a Rubin (18), donde se propone la hipótesis de que los varones homosexuales prefieren parejas mayores cuando son jóvenes y a parejas con menor edad que ellos cuando envejecen, y que esta diferencia se incrementa a medida que cumplen años; las lesbianas elegirán parejas mayores y esta diferencia se mantendrá a lo largo del ciclo vital femenino.

De otro lado, al analizar los resultados del uso de lubricantes en las prácticas sexuales, el 42,8\% de los homosexuales manifestó que sí utiliza lubricantes en sus prácticas sexuales, el $41,1 \%$ no los utiliza y el $16,1 \%$ a veces los usa, evidenciándose con mayor porcentaje las personas que sí los utilizan, no siendo muy significativa la diferencia con las personas que no los utilizan y dando menor porcentaje a las personas que los utilizan ocasionalmente.

Al comparar los resultados con el estudio de Dezzutti (19), que buscaba concluir si los hombres y mujeres homosexuales hacían uso de lubricantes o no y su relación con la adquisición de infecciones de transmisión sexual, los resultados mostraron que el $26,2 \%$ afirmó que habían empleado lubricantes en sus relaciones sexuales receptivas, el uso de lubricantes fue menos habitual entre personas de origen afroamericano con un $38,5 \%$; y no se hacía uso de ellos en personas de origen hispano en un $35,3 \%$, pues se consideró que el no uso de ellos o el uso ocasional era a consecuencia de que los lubricantes no estaban regulados y ponían en riesgo su salud.

Al respecto, Dezzutti (19) afirma que "los lubricantes pueden aumentar el riesgo de adquirir infecciones de transmisión sexual" evidenciando que no todos los homosexuales hacen uso de los lubricantes, por diferentes razones asociadas o no a la adquisición de infección de transmisión sexual.

Al analizar los resultados del uso de sustancias psicoactivas, se puede evidenciar que el $70 \%$ de la población no hace uso de sustancias psicoactivas en sus relaciones sexuales, mientras que el 13,9\% sí lo hace. En comparación con el estudio de Cáceres (20) que menciona como la respuesta más frecuente entre los encuestados "a lo que venga, alcohol, drogas y vulnerabilidad sexual", se puede evidenciar que no todos los homosexuales hacen uso de estas sustancias en sus prácticas sexuales, muchos son conscientes de la afección que trae para su salud sexual y reproductiva, mientras otros no le prestan atención a las consecuencias.

El estudio de Cáceres (20) muestra que con el uso de sustancias psicoactivas existe aumento en el número de parejas sexuales y una mayor frecuencia de prácticas de riesgo con todas las parejas sexuales. De todos los homosexuales presentes en el estudio, un elevado porcentaje, correspondiente al 75\%, mantuvo relaciones sexuales bajo los efectos de sustancias 
psicoactivas y el 32,9\% intercambió sustancias psicoactivas por sexo

\section{Desarrollo en salud sexual y reproductiva}

El $91,1 \%$ de los homosexuales manifestó no haber presentado ninguna infección de transmisión sexual a lo largo de su vida, mientras que el $8,9 \%$ de la población sí lo ha presentado; sólo uno de los encuestados presenta el virus de inmunodeficiencia humana (VIH). Se evidencia que no toda la población padece o ha padecido alguna infección de transmisión sexual, tal como se muestra en la Estrategia de Información, Educación y Comunicación en el Abordaje de las Infecciones de Transmisión Sexual y el VIH, elaborada por el Ministerio de Salud en El Salvador (21), donde se afirma que las parejas homosexuales que tienen contacto sexual están más propensas a adquirir estas enfermedades, que en el caso de las relaciones heterosexuales.

Los primeros y la mayoría de casos de enfermedades adquiridas mediante contacto sexual se han presentado en países con mayor grado de promiscuidad y homosexualidad. Las cifras obtenidas renuevan preocupación ante los hallazgos, sobre todo con el grupo de lesbianas que, por las cuestiones de género, ha sido considerado tradicionalmente como un grupo de bajo riesgo frente al VIH y otras infecciones de transmisión sexual (ITS), sin serlo (13).

Cabe resaltar que a la hora de encuestar no todas las personas son abiertas para manifestar que poseen una afección en su salud y muchas veces, por temor, se prefiere manejar el tema con discreción. Así mismo, en la actualidad se han presentado tantos casos que muchos homosexuales toman conciencia de sus actos y mantienen una sexualidad responsable por lo que, a lo largo de su vida, disfrutan plenamente de su sexualidad sin ningún tipo de riesgo (13).

Al realizar el análisis de si los homosexuales que presentaron infecciones de transmisión sexual (ITS) recibieron tratamiento completo, se evidencia que el $91,1 \%$ de la población no ha presentado ningún tipo de infecciones de transmisión sexual y, por lo tanto, no ha recibido tratamiento; el otro $8,9 \%$ es la población que sí las ha presentado y, en su totalidad, ha recibido tratamiento completo.

Esta situación entra en contraste con el estudio de
Moreno (6), donde los participantes que habían padecido alguna infección de transmisión sexual equivalían solamente al $59,8 \%$ y habían tenido tratamiento, mostrando que no toda la población que padece este tipo de afecciones se somete a tratamiento.

Al analizar los resultados sobre si la población homosexual utilizó o no preservativo en su primera relación sexual, se puede evidenciar que el $56,7 \%$ sí lo utilizó, mientras que el $43,3 \%$ de la población no lo utilizó, indicando que no toda la población tiene estas medidas preventivas desde la primera relación sexual, convirtiéndose en una práctica de riesgo para la adquisición de infecciones de transmisión sexual.

Esta situación se evidencia en los resultados de la encuesta realizada por el Ministerio de Salud de São Paulo (22), donde se muestra que de 108 jóvenes que se consideran gais y lesbianas el $20 \%$ afirmó que el uso de preservativos en la primera relación sexual no ocurrió y el $43,7 \%$, correspondiente a mujeres, indicó que no usó preservativo en su primera relación sexual ni en sus siguientes relaciones sexuales con mujeres $u$ hombres en algunos casos.

Al hacer el análisis de si los homosexuales tienen conocimiento sobre el cáncer genital, se puede evidenciar que el $54,4 \%$ de la población sí tiene conocimiento sobre el tema, mientras que el $45,6 \%$ de la población no lo posee; también se observa que no toda la población analizada tiene conocimiento acerca del cáncer genital, considerado un problema de salud pública que involucra directamente a este grupo específico de la población, hecho que se convierte en una conducta de riesgo para la salud de los homosexuales, tal como se muestra en el estudio de Moreno (6), que obtuvo como respuesta mayoritaria que sí se tiene conocimiento acerca del cáncer genital, en un $67,3 \%$.

Las lesbianas corren mayor riesgo que las heterosexuales de desarrollar cáncer de mama por cuanto no han estado embarazadas, en comparación con las heterosexuales dado que durante el embarazo se liberan ciertas hormonas que tendrían un efecto preventivo con respecto al cáncer (5).

Para el cáncer cervicouterino se confirma que los factores de riesgo se refieren al posible contagio con el virus del papiloma humano (VPH), que puede transmitirse de mujer a mujer en la relación sexual, 
ISSN 1794-9831

E-ISSN 2322-7028

Vol. 12 No. 1

Ene - Jun 2015

Cúcuta, Colombia constituyéndose en un grave problema de salud pública del cual se presentan 38.000 casos nuevos anuales, ocupando el segundo lugar entre las causas de mortalidad (5).

\section{CONCLUSIONES}

Al caracterizar sociodemográficamente la población, se encontró que predominaron los gais, no siendo muy relevante la diferencia con las lesbianas. De ellos, el estado civil de mayor porcentaje fue soltero, seguido de unión libre; la edad que predominó fue de 24 a 29 años, sin una diferencia significativa; el nivel educativo que más se presentó fue secundaria completa y un bajo porcentaje fue de profesionales.

En cuanto a la información sobre las experiencias sexuales, la primera experiencia física con una persona de su misma orientación sexual fue sexo, la mayoría de población inicio su vida sexual entre las edades de 14 a 19 años, demostrando que iniciaron su vida sexual muy temprano, siendo este un factor de riesgo.

Parte de la población ha tenido de 11 a 20 y de 21 a 30 compañeros sexuales a lo largo de su vida, lo cual deja ver la alta tasa de promiscuidad presente; se evidencia también que en los últimos 6 meses más de la mitad de la población ha tenido relaciones sexuales con personas ocasionales, pero la misma cantidad de población manifiesta que el mayor temor relacionado con el sexo es contraer una enfermedad, lo cual indica que los homosexuales son conscientes de los riesgos que tienen en su vida sexual, pero de igual forma no realizan acciones preventivas para obtener el bienestar total en su salud sexual y reproductiva.

Frente a las actitudes de los homosexuales, se evidenció que las primeras personas que conocieron su orientación sexual fueron los amigos de los encuestados y no la familia, viéndose afectada su salud mental en cuanto a los lazos afectivos de aceptación y confianza con su entorno más cercano. Otro dato importante es que la mayor parte de la población quiso volver a tener una experiencia sexual por gusto y/o placer, lo cual se considera una conducta de riesgo para su salud sexual y reproductiva por cuanto se realiza pensando sólo en gusto y/o placer y olvidando las consecuencias negativas que traería para la salud.

En cuanto a las prácticas, se demostró que más de la mitad de la población conoce a sus parejas sexuales por amigos, constituyéndose en un factor de riesgo para su salud sexual y reproductiva, puesto que se desconoce el pasado sexual de dichas personas con las cuales se llevan a cabo relaciones sexuales; otra práctica de riesgo fue la del sexo oral que realizan con la mayoría de los contactos sexuales, que ante la presencia de laceraciones $\mathrm{u}$ afecciones en la piel, posibilitan la adquisición de infecciones de transmisión sexual, afectando la salud sexual y reproductiva.

La población que ha presentado infecciones de transmisión sexual a lo largo de su vida, en su totalidad ha recibido tratamiento completo, indicando la acción oportuna de los servicios de salud para tratar este tipo de problemas en la población homosexual.

Además, un factor de riesgo que se identificó en la investigación radica en la posibilidad de adquirir una ITS en la primera relación sexual, a partir de la no utilización de preservativo en el primer encuentro sexual, debido a la desinformación o información errada que posee la población acerca de los métodos de protección, desde su primera relación sexual.

En cuanto a la información que poseen los homosexuales acerca del cáncer genital, más de la mitad de la población sabe qué es el cáncer genital, siendo parcialmente equitativa la respuesta con quienes no saben qué es, demostrando que un problema de salud pública tan importante y de tan alta incidencia no es conocido por la totalidad de la población que más se ve involucrada. 


\section{REFERENCIAS BIBLIOGRÁFICAS}

1. Parejas Homosexuales. Definición de sexualidad en adolescentes homosexuales. [Internet]. 2012 [consultado 7 de septiembre de 2013]. Disponible en: http://www.parejashomosexuales.com/relaciones/definicion-de-sexualidaden-adolescentes-homosexuales.php

2. Fondo de Población de las Naciones Unidas (UNFPA). Salud sexual y reproductiva Promoviendo la salud para el futuro. [Internet]. [consultado 21 de marzo de 2014]. Disponible en: http://www.unfpa.org.mx/salud\%20 sexual\%20y\%20reproductiva.php

3. Calvis M. Prácticas homosexuales. [Internet]. [consultado 7 de septiembre de 2013]. Disponible en: http://www. aciprensa.com/Familia/homosex-practic.htm

4. República de Colombia. Ministerio de Salud. Resolución No. 008430 de 1993, octubre 4, por la cual se establecen las normas técnicas y administrativas para la investigación en salud. [Internet]. 1993 [consultado 21 de marzo de 2014]. Disponible en: http://www.urosario.edu.co/EMCS/Documentos/investigacion/resolucion_008430_1993/

5. Gonzáles-Guadamarra J, Toro-Alfonso J. El significado de la experiencia de la aceptación de la orientación sexual homosexual desde la memoria de un grupo de hombres adultos puertorriqueños. Eureka [Internet]. 2012 [consultado 15 de abril de 2014]; 9(2): 158-170. Disponible en: http://www.psicoeureka.com.py/sites/default/ files/articulos/eureka-9-2-12-10.pdf

6. Moreno-Gutiérrez NI. Situación de salud sexual y reproductiva, hombres y mujeres homosexuales Hospital María Auxiliadora 2006. Rev Per Obst Enf. [Internet]. 2007 [consultado 10 de octubre de 2014]; 3(1):2-16. Disponible en: http://revistas.concytec.gob.pe/scielo.php?pid=S1816-77132007000100002\&script=sci_arttext

7. Federación Estatal de Lesbianas y Gais (FELG) - Durex España. Conducta sexual entre homosexuales adolescentes. [Internet]. España: FELG - Durex; 2002 [consultado 9 de octubre 2013].Disponible en: http://www.sidastudi.org/ resources/inmagic-img/dd0407.pdf

8. Ortiz-Hernández L, García-Torres M. Opresión internalizada y prácticas sexuales de riesgo en varones homo- y bi-sexuales de México. Rev Saúde Pública [Internet]. 2005 [consultado 15 de abril de 2014]; 36(6):956-964. Disponible en: http://www.scielosp.org/pdf/rsp/v39n6/26992.pdf

9. Cañejas S, Pérez J, Noguer I, Villamil F, García ML, De la Fuente L. et al. Conductas sexuales de riesgo y prevalencia de infecciones por VIH en hombres con prácticas homosexuales y bisexuales en la comunidad de Madrid. Rev Esp Salud Pública [Internet]. 2000 [consultado 15 de abril 2014]; 74(1): 25-32. Disponible en: http:// scielo.isciii.es/scielo.php?pid=S1135-57272000000100004\&script=sci_arttext

10. Moral-De la Rubia J. Conducta homosexual: una perspectiva integradora biopsicosocial. Revista Electrónica de Psicología Iztacala. [Internet]. 2009 [consultado 15 de abril 2014]; 12(3):44-69. Disponible en: http://www. revistas.unam.mx/index.php/repi/article/view/15472

11. Nader-Mora L, Villegas-Palacio M, Alejao-Riveros M, Eraso-López de Mesa A, Salazar-Santos G. Preguntas que hacen los adolescentes de 14 a 22 años. [Internet]. Bayer Países Andinos; 2012 [consultado 15 de abril de 2014]; p. 1-30. Disponible en: http://www.bayerandina.com/descargas/libro_sexualidad.pdf

12. Irvin-Jaén A, Hidalgo-Abellé A. Mitos y realidades acerca de la homosexualidad en el siglo XXI. [Internet]. Valencia: Clínica Psicológica V. Mars - Sueca; 2010 [actualizado de 8 marzo de 2010; consultado 15 de abril de 2014]. Disponible en: http://www.psicologia-online.com/monografias/7/mitos_homosexualidad. shtml

13. Estudiosexual.com. Resultados y conclusiones 2010. [Internet]. 2010 [consultado 15 de abril de 2014]. Disponible en: http://www.estudiosexual.com/results.aspx

14. Eighner L. Homosexualidad: el mito romántico y la trágica realidad. [Internet]. 2014 [consultado 15 de abril de 2014]. Disponible en: http://www.accionfamilia.org/temas-polemicos/ homosexualidad/homosexualidad-el-mitoromantico-y-la-tragica-realidad/\#_ftn9_8841

15. De La Cruz C. Taller adolescente sexualidad homosexualidad. [Internet]. Madrid: AMPAP; 2013 [actualizado 25 Ene 2013; consultado 15 de abril de 2014]. Disponible en: http://www.ampap.es/docencia/ pdf/2013_taller_ adolescente_sexualidad_homosexualidad.pdf

16. Family Research Institute, Inc. (Instituto de Investigaciones para la Familia). Consecuencias médicas de lo que hacen los homosexuales. Providafamilia.org. [Internet]. Washington; 2013 [consultado 15 de abril 2014]. Disponible en: http://providafamilia.org/doc.php?doc=doc49291

17. Gómez J. Análisis evolutivo de la diferencia de edad en la elección de pareja en España durante el período 1976 2006. Rev Psicol Soc [Internet]. 2011 [citado 15 Abr 2014]; 26(1):73-89. Disponible en: http:// webdeptos.uma. es/psicologiasocial/luisgomez/analisis_evolutivo_de_la_diferencia_de_edad_en_la_eleccion_de_pareja.pdf

18. Rubín R. Notas para una teoría radical de la sexualidad. Bibli Virtual Cienc Soc [Internet]. 2009 [consultado 21 de octubre de 2013]; 1(1):1-59. Disponible en: http://webs.uvigo.es/xenero/profesorado/beatriz_suarez/rubin.pdf 
19. Dezzutti C. [Internet]. Barcelona: GTT; 2010 [actualizado 5 Mar 2010; consultado 15 de abril de 2014]. Disponible en: http://gtt-vih.org/book/print/5388

20. Cáceres C. Unidad de salud, sexualidad y desarrollo humano. 1 ed. [Internet]. Lima: UNODC; 2010 [18 de septiembre de 2010; [consultado 15 de abril de 2014]. Disponible en: http:/www.unodc.org/documents/ peruandecuador/Publicaciones/A_lo_que_venga.pdf

21. Ministerio de Salud. Estrategia de información, educación y comunicación en el abordaje de las ITS y VIH. $1^{\text {a }}$ ed. [Internet]. El Salvador: Ministerio de Salud; 2012 [consultado el 15 de abril de 2014]. Disponible en: http://asp. salud.gob.sv/regulacion/pdf/estrategias/estrategia_iec_its_vih.pdf

22. Ministerio de Salud de São Paulo. Encuesta los homosexuales jóvenes no siempre usan condones en las relaciones sexuales. [Internet]. 2013 [consultado 15 de abril de 2014]. Disponible en: http://www.isaude.net/ es/noticia/35618/foto-de-la-salud/42-de-los-hombres-homosexuales-jovenes-no-siempre-usan-condones-en-lasrelaciones-sexuales

\section{BIBLIOGRAFÍA}

American Psychological Association. Orientación sexual y homosexualidad. [Internet]. Washington: APA; 2010 [consultado 4 de septiembre de 2013]. Disponible en: http://www. apa.org/centrodeapoyo/sexual.aspx

Casabona J. Casi la mitad de gays tienen relaciones sexuales sin protección. Efesalud.com [Internet]. Madrid; 2013 [actualizado 4 Jul 2013; consultado 15 de abril de 2014]. Disponible en: http://www.efesalud.com/noticias/casi-la-mitadde-gays-tienen-relaciones-sexuales-ocasionales-sin-proteccion

Centro de Especialistas de Medicina de España. Temas de sexualidad - El tamaño del pene es importante. [Internet]. Bogotá: MD; 2012 [consultado 15 de abril de 2014]. Disponible en: http://www.medicoensudomicilio.com/pages/temasde-sexualidad/el-tamano-del-pene-es-importante.html

Centro de Psicología Sexología y Psicopedagogía. [Internet]. Bogotá: Aide Psicología; 2012 [actualizado 17 Feb 2012; citado 15 Abr 2014]. Disponible en: http://aidepsicologia.wordpress.com/

Instituto Nacional de Estadística y Geografía México. Estadísticas a propósito del día mundial de la lucha contra el sida [Internet]. 2011 [consultado 4 de septiembre de 2013]. Disponible en: http://www.inegi.org.mx/inegi/contenidos/espanol/ prensa/contenidos/estadisticas/2011/sida11 asp?s $=$ inegi\&c $=2819 \&$ ep $=78$

Instituto Valenciano de Fertilidad, Sexualidad y Relaciones Familiares. Favorece el sida la Homosexualidad [Internet]. 2009 [consultado 28 de agosto de 2013]. Disponible en: http://www.sinsida.com/montador.php?tipo=homosexualidad

Instituto de Psicología y Sexología Impronta. Psicología y Sexología. Confusión de Orientación Sexual (Homosexualidad). [Internet]. 2009 [consultado 28 de agosto de 2013]. Disponible en: http://www.psico-impronta.com/Psicologia-ySexologia/Confusion-de-orientaci\%C3\%B3n-sexual-Homosexualidad.axd

Organización Mundial de la Salud. Sexualidad 2010 [Internet]. México: OMS; 2010 [actualizado 25 de agosto de 2010; consultado 8 agosto de 2013]. Disponible en: http://search. who.int/ search?q=sexualidad\&ie=utf8\&site=defau 1t_collection\&client $=$ _es\&proxystylesheet=_es\&output=xml_no_dtd\&oe=utf8

Programa Conjunto de las Naciones Unidas sobre el VIH/SIDA (ONUSIDA). Informe Epidemia 2008. Cifras del mundo. [Internet]. Ginebra: ONUSIDA; 2008 [actualizado 8 de agosto de 2008; consultado 4 de septiembre de 2013]. Disponible en: http://www.censida.salud.gob.mx/ descargas/cifras_mundo/informe_epidemia2008.pdf

Saavedra C. El informe Kinsey. Rev Indice [Internet]. 2006 [consultado 10 de octubre de 2013]; 1(15):20-22. Disponible en: http://www.revistaindice.com/numero15/

Vans Pritchard E. Sexual inversion among the azande. Am Anthrop New Series [Internet]. 1970 [consultado 28 de agosto de 2013]; 72(6):1428-1434. Disponible en: http://disciplinas.stoa.usp.br/pluginfile.php/18137/mod_resource/content/ 1/ AzandeEvansPritchard.pdf 